Recent was defined as admission within the last 3 months prior to the interview. The interviews were conducted in patients' homes. The sample comprised 12 males (63\%), 7 (37\%) females. The mean age for males and females was 70 years. The data was coded and grouped into 5 categories. The findings for the categories: "Going to hospital" and "Discharge from hospital" are presented.

Findings COPD patients have much experience in managing an exacerbation of their condition and can recognise significant changes in their health status requiring urgent hospitalisation. The findings for "Going to hospital" include: who patients rely on to make the decision, how the decision is made/or avoided and their emotive experiences of going to hospital. Patient experiences around "Discharge from hospital" include: consultation, confusion, timeliness and (lack of) streamlining in the discharge process. Patient narratives will be presented.

Conclusion Decision-making about going to hospital and the subsequent discharge process can be viewed as the beginning and end points in the hospitalisation journey for COPD patients. Patient experiences about these points in the journey give key insights into decision making, quality care and identify benchmarks for future reviews of service provision to COPD patients.

\section{P81 LUNG TRANSPLANTATION IN ALPHA-1-ANTITRYPSIN DEFICIENCY}

doi:10.1136/thoraxjnl-2012-202678.323

HM Stone, R Edgar, RA Stockley. University Hospital Birmingham NHS Foundation Trust, Birmingham, UK

Background Lung transplantation is an option for a proportion of patients with end-stage lung disease who deteriorate despite optimal medical therapy. In patients with alpha-1-antitrypsin deficiency (A1ATD), survival benefit has been demonstrated following transplantation. Patient selection is often subjective and the effects of transplantation on Health Related Quality of Life (HROOL) in A1ATD are unknown. The aims of the current study were to determine objective measures influencing patient selection, survival benefit and health status following lung transplantation in A1ATD patients.

Methods Between 1996 and 2011, patients with A1ATD (PiZ) who had undergone lung transplantation were identified from the UK A1ATD registry. Lung function parameters, including rates of decline pre-transplant, were compared to matched subjects who did not undergo transplantation (matched for sex, age, smoking history and $\mathrm{FEV}_{1}$ ). HROOL, assessed using the St George's Respiratory Questionnaire (SGRQ), was measured pre and post-transplant together with mortality data.

Results Patients who underwent transplantation $(n=32)$ had significantly worse HRQOL than patients who did not in all domains of the SGRQ (total score 64.2; $\mathrm{SE} \pm 2.5$ vs. $55.3 \pm 2.04, \mathrm{p}=0.008$ ). Markers of gas transfer (TLCO and KCO) were both significantly lower in the transplantation group $(33.5 \% \pm 3.0$ and $41.0 \% \pm 3.9$ predicted respectively, compared to $50.6 \% \pm .69$ and $57.58 \% \pm 3.1$ predicted in the non-transplant group $p<0.001 \& p=0.001)$. There were no differences in the rate of lung function decline compared to non-transplanted patients.

Sixteen transplant patients died in the follow up period ( 6 perioperatively). Figure 1 summarises the Kaplan-Meier survival curve following transplantation. There was no relationship to pretransplant physiology. Once a survival time of 5 years had been reached, mortality was due to causes unrelated to A1ATD or the transplant.

There was a significant improvement in spirometry $(23.94 \pm 1.0$ vs. $92.73 \pm 9.2, p=0.002, N=16)$ and all domains of the SGRQ post lung transplantation, including the total score $(64.2 \pm 2.5$ vs. 15.6 $\pm 4.9, \mathrm{p}=0.002$ ).

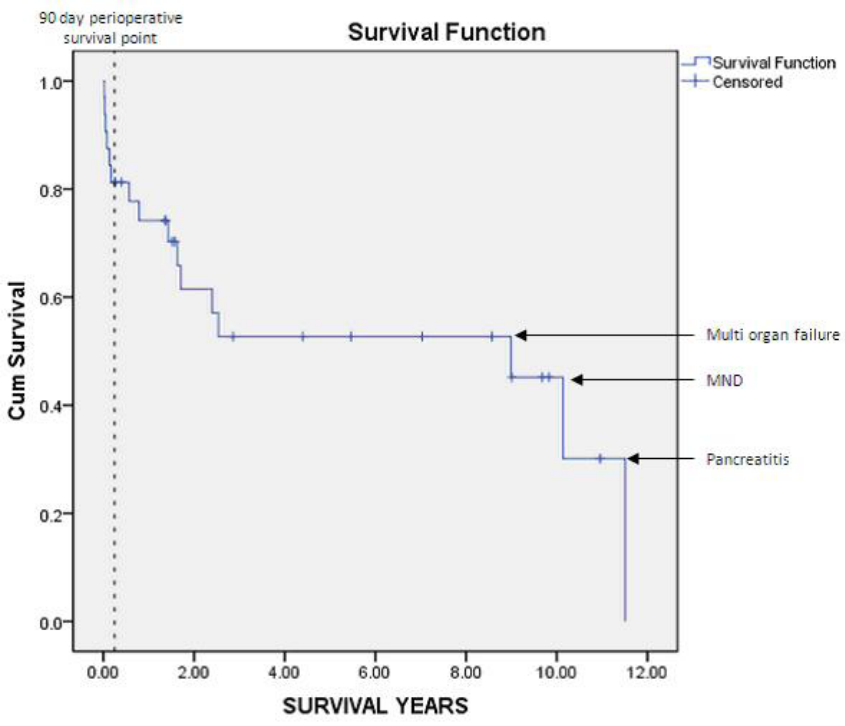

Figure 1 Kaplan-M eier survival curve following transplantation with cause of death post 5 year survival.

\section{Abstract P81 Figure 1}

Conclusion Patients who underwent lung transplantation had worse gas transfer parameters pre transplant compared to the otherwise matched A1ATD patients who did not. Lung transplantation in patients with end-stage lung disease secondary to A1ATD significantly improved HRQOL.

\section{P82 EFFECT OF HOSPITALISATION FOR ACUTE EXACERBATIONS OF COPD ON SUBSEQUENT QUALITY OF LIFE}

doi:10.1136/thoraxjnl-2012-202678.324

'J Steer, ${ }^{2} \mathrm{GJ}$ Gibson, 'SC Bourke North. 'Tyneside General Hospital, North Shields, United Kingdom; ${ }^{2}$ Newcastle University, Newcastle-upon-Tyne, United Kingdom

Background The longitudinal change in quality of life ( $\mathrm{OoL}$ ) following hospitalisation for acute exacerbations of COPD (AECOPD) is uncertain. Current guidelines suggest that non-invasive ventilation (NIV) should not be considered if there is no "potential for recovery to QoL acceptable to the patient",[1] but the expected QoL recovery in this population has not been reported.

Methods 183 patients ( 82 treated with NIV) with AECOPD surviving to hospital discharge were identified prospectively. QoL questionnaires were completed at hospital discharge and at four predefined intervals during the year following discharge, however, for brevity, only the results of the St. George's Respiratory Questionnaire (SGRQ) are reported. Baseline clinical information and subsequent mortality and readmission over 12 months were recorded. Longitudinal $\mathrm{QoL}$ was summarised by calculating the mean change in SGRQ (mean change $<0$ indicates a QoL improvement during follow-up), with the minimal clinically important SGRQ change taken as \pm 4 .

Results Mean (SD) age was 69.0 (9.0) years and most patients: were female (58.5\%); had severe airflow obstruction (mean (SD) $\mathrm{FEV}_{1} 40.2$ (17.3) \% predicted); and were of normal weight (mean (SD) BMI $26.2(7.0) \mathrm{kgm}^{-2}$ ). The 12-month readmission and mortality rate were $71 \%$ and $18 \%$ respectively.

7 patients failed to attend follow-up after discharge and were excluded from analysis. For both patient groups, self-reported respiratory symptoms (SGRO Symptoms) improved by a clinically important amount during follow-up. For the total population, average overall QoL (SGRO total) improved during follow-up (mean (SD) change in SGRQ total $=-2.47(13.0)$ ), but not by a clinically important amount. Patients not treated with NIV experienced both 